\title{
Measuring Global and Local Spatial Correspondence Using Information Theory
}

\author{
F. Bello and A.C.F. Colchester \\ Neurosciences Medical Image Analysis Group, Kent Institute of Medicine and Health \\ Sciences, University of Kent at Canterbury, Canterbury Kent CT2 7NT, UK \\ f.bello@ukc.ac.uk
}

\begin{abstract}
The evaluation of spatial correspondence between binary objects resulting from a segmentation step performed by two different observers or methods is a critical part of the validation of a segmentation criterion or technique. Several global measures of correspondence have been previously proposed, but all of them assume a one-to-one correspondence between objects, thus failing to address local problems such as the splitting of an object by one of the observers. Moreover, such global measures do not distinguish between the reference and the observed objects and most of them lack a solid theoretical foundation. In this paper, we introduce a set of spatial correspondence indices that can evaluate global (many-to-many), local (many-to-one) and individual (one-to-one) spatial correspondence between observed and reference objects and vice versa. The proposed measures, derived from applying information theory concepts to the problem of spatial correspondence, are shown to be well-behaved and suitable to be used in medical imaging applications.
\end{abstract}

\section{Introduction}

One of the central problems in the area of medical image computing is the validation of results from a segmentation algorithm. Such validation or comparison always requires at certain levels of abstraction the recognition of similarities or differences between two representations, one representation being considered as the current observation, and the other as some reference model or ground truth. Quantifying these similarities by developing suitable similarity measures is often quite difficult.

The concept of pattern similarity is key to many statistical, syntactic and neural pattern recognition techniques [1]. Within the area of medical imaging various measures have been used to assess the similarity or agreement between segmentation algorithms and human observers [2] [3] [4] and between observers [5], but they all have been used as global measures of similarity with some elaborated on an empirical basis without adequate theoretical justification. The spatial correspondence indices introduced here are based on information theory, which has been successfully used to register medical images by maximisation of mutual information [6] [7]. In fact, the main difference between those methods and the concept introduced here is that registration methods assume that the majority of the underlying objects which generated both representations were the same. 
The methods then seek the transformation that will maximise spatial correspondence, regardless of the actual values of the objective function. In contrast, we are attempting to directly measure the spatial correspondence and utilise the measures to analyse details of the similarities and differences.

Our immediate motivation is the comparison and validation of lesions segmented by two different observers or segmentation methods on the same, i.e. perfectly registered, magnetic resonance (MR) brain scans of multiple sclerosis (MS) patients. Such comparison must take into account the inherent difference between identifying the presence of a lesion and the accurate placement of a boundary around it. Provision of global and local quantitative measures of agreement can significantly improve the understanding of the lesion identification and delineation process, as well as simplify the analysis and validation of segmentation results in large numbers of scans.

\section{Spatial Correspondence}

Let $\boldsymbol{X}$ be the set of objects $X_{k}, k=1 \ldots, K$ in the reference model and $\boldsymbol{Y}$ be the set of objects $Y_{j}, j=1, \ldots, J$ in the current observation. Consider $\boldsymbol{X}$ and $\boldsymbol{Y}$ as two different partitions of the same image and assume that there are $Q$ locations equally spaced in $G$, a lattice extending over the area covered by both partitions. Each point in $\boldsymbol{G}$ can be identified in relation to the objects in $\boldsymbol{X}$ and the objects in $\boldsymbol{Y}$. Let $f_{k j}$ be the number of lattice points to be found in $X_{k} \cap Y_{j}$ with $\sum_{k=1}^{K} \sum_{j=1}^{J} f_{k j}=Q$. Then, $P_{X Y}(k, j)=f_{k j} / Q$ is the probability that a point in $\boldsymbol{G}$ falls in objects $X_{k}$ and $Y_{j}$. Similarly, $P_{X}(k)=f_{k} / Q$ and $P_{Y}(j)=f_{j} / Q$ give the probability that a point in $\boldsymbol{G}$ will fall in object $X_{k}$ or object $Y_{j}$, respectively.

An object $Y_{j}$ present in the observation is said to correspond to the object $X_{k}$ in the reference model if and only if the set of all lattice points $\left\{y_{j 1}, \ldots, y_{j L}\right\} \in Y_{j}$ is the same as the set of all lattice points $\left\{x_{k 1}, \ldots, x_{k M}\right\} \in X_{K}$. As each of the lattice points $y_{j l}$ and $x_{k m}$ has a unique spatial location, the correspondence between objects $Y_{j}$ and $X_{k}$ implies not only that $P_{Y}(j)=P_{X}(k)$, but also that the space occupied by $\left\{y_{j 1}, \ldots, y_{j L}\right\}$ must be the same as that occupied by $\left\{x_{k 1}, \ldots, x_{k M}\right\}$. It is only when these two conditions are met that both identification and boundary delineation errors can be avoided.

Of the measures used in the analysis of MS segmentations, the area error and the correlation coefficients [4] [5] only evaluate the agreement in terms of the total number of elements in each segmentation, thus making a more detailed analysis of the segmentation errors impossible. The similarity index [2] and overlap index [3] consider the total number of elements and the total overlap to give an overall measure of agreement, but they are still global measures and therefore not capable of discriminating between the different types of segmentation errors.

To address local spatial correspondence we create a joint frequency distribution based on the above definitions by means of a Correspondence matrix $\boldsymbol{O}$. This matrix is similar in concept to the co-occurrence matrix widely used in 
texture analysis. Assuming that all the objects in $\boldsymbol{X}$ and $\boldsymbol{Y}$ have been uniquely labelled, the columns of $\boldsymbol{O}$ correspond to the objects $Y_{j}, j=1, \ldots, J$ in the observation and the rows to the objects $X_{k}, k=1 \ldots, K$ in the reference. Each entry $o_{k j}$ in $\boldsymbol{O}$ indicates the number of lattice points common to objects $X_{k}$ and $Y_{j}$, i.e. $X_{k} \cap Y_{j}$. The background in each partition, $\overline{\boldsymbol{X}}$ and $\overline{\boldsymbol{Y}}$, is treated as a separate object to allow the computation of a valid joint probability distribution from $\boldsymbol{O}$. The marginal probabilities for each object, $P_{X}(k)$ and $P_{Y}(j)$, can then be computed by adding all entries across the rows or columns. Various other global and local measures such as those to be introduced in the next section can be directly computed from $\boldsymbol{O}$. As $P_{X Y}(k, j), P_{X}(k)$ and $P_{Y}(j)$ are dependent on the density of points in $G$, a relatively dense pattern of points must be used for a reliable estimation.

\section{Theoretical Framework}

If we interpret $\boldsymbol{X}$ as the input into a noisy discrete channel and $\boldsymbol{Y}$ as the output from the channel, using the information theory concepts introduced by Shannon [8] we know that the information provided about the event $X_{k}$ occurring at the source by the occurrence of event $Y_{j}$ at the output is given by their mutual information $I_{X ; Y}(k, j)=\log \left(P_{X \mid Y}(k, j) / P_{X}(k)\right)$. A special case of $I_{X ; Y}$ is when knowledge of the output uniquely determines the input, i.e. $P_{X \mid Y}(k, j)=1$. In this case $I_{X ; Y}$ is known as the self information of $X_{k}$ or $Y_{j}$ (denoted by $I_{X}(k)$ and $I_{Y}(j)$ ). This situation occurs if the discrete channel is noiseless and there is full agreement between $\boldsymbol{Y}$ and $\boldsymbol{X}$. By considering such a situation, it is possible to define $C_{j k}$, a measure of spatial correspondence between object $Y_{j}$ and object $X_{k}$, as the ratio of the average mutual information between the objects, $I\left(X_{k} ; Y_{j}\right)$, to the average self information or entropy, $H\left(X_{k}\right)$, of the reference object:

$$
C_{j k}=\frac{I\left(X_{k} ; Y_{j}\right)}{H\left(X_{k}\right)}=\frac{P_{X Y}(k, j)}{P_{X}(k)} \cdot \frac{I_{X ; Y}(k, j)}{I_{X}(k)}
$$

Similarly, if $\boldsymbol{Y}$ is considered as the reference, $C_{k j}$ measures the correspondence between the objects $X_{k}$ and $Y_{j}$ :

$$
C_{k j}=\frac{I\left(X_{k} ; Y_{j}\right)}{H\left(Y_{j}\right)}=\frac{P_{X Y}(k, j)}{P_{Y}(j)} \cdot \frac{I_{X ; Y}(k, j)}{I_{Y}(j)}
$$

$C_{j k}=C_{k j}$ if $Y_{j}$ and $X_{k}$ have the same number of elements or lattice points, regardless of their spatial location.

Using the average mutual information between $Y_{j}$ and $X_{k}, k=1, \ldots, K$ it is also possible to compute $C_{j}$, a measure of the local spatial correspondence between $Y_{j}$ and all the objects in $\boldsymbol{X}$. Equally, the local spatial correspondence between $X_{k}$ and all the objects in $\boldsymbol{Y}$ can be estimated by computing $C_{k}$ :

$$
C_{j}=\frac{I\left(\boldsymbol{X} ; Y_{j}\right)}{H\left(Y_{j}\right)} \quad C_{k}=\frac{I\left(X_{k} ; \boldsymbol{Y}\right)}{H\left(X_{k}\right)}
$$


From the definition of average mutual information we can deduce that $C_{j}$ and $C_{k}$ effectively measure the degree of spatial correspondence between the selected object and all those objects in the alternate representation with which it shares at least one element. Furthermore, these measures obey the following relationship:

$$
C_{j}=\sum_{k=1}^{K} C_{k j} \quad C_{k}=\sum_{j=1}^{J} C_{j k}
$$

The above measures estimate the spatial correspondence between an individual object and those with which it has common elements. A global measure of spatial correspondence between the observation $\boldsymbol{Y}$ and the reference $\boldsymbol{X}$ can be obtained by dividing the average total mutual information $I(\boldsymbol{X} ; \boldsymbol{Y})$ by the average total self information or entropy of $\boldsymbol{X}, H(\boldsymbol{X})$.

$$
C_{\boldsymbol{Y}}=\frac{I(\boldsymbol{X} ; \boldsymbol{Y})}{H(\boldsymbol{X})}=\frac{\sum_{k=1}^{K} \sum_{j=1}^{J} P_{X Y}(k, j) \cdot \log \frac{P_{X Y}(k, j)}{P_{X}(k) P_{Y}(j)}}{\sum_{k=1}^{K} P_{X}(k) \cdot \log \frac{1}{P_{X}(k)}}
$$

Similarly, if $\boldsymbol{Y}$ is considered as the reference, $C_{\boldsymbol{X}}$ estimates the global spatial correspondence between $\boldsymbol{X}$ and $\boldsymbol{Y}$ :

$$
C_{\boldsymbol{X}}=\frac{I(\boldsymbol{X} ; \boldsymbol{Y})}{H(\boldsymbol{Y})}=\frac{\sum_{k=1}^{K} \sum_{j=1}^{J} P_{X Y}(k, j) \cdot \log \frac{P_{X Y}(k, j)}{P_{\boldsymbol{X}}(k) P_{Y}(j)}}{\sum_{j=1}^{J} P_{Y}(j) \cdot \log \frac{1}{P_{Y}(j)}}
$$

\section{Experiments}

Three types of objects were used to study the new measures: single synthetic 2-D objects, multiple synthetic 2-D objects and 3-D MS lesions segmented by two different observers (Fig. 1). In all the experiments a complement area error measure $(\operatorname{Err} A=1-2 *|b-c| /(b+c))$, the overlap index $(O v e r=a /(b+c-a))$ and the similarity index $(\operatorname{Sim}=2 * a /(b+c))$ were also computed. The size of the objects $(b, c)$ and the overlap $(a)$ are expressed in number of lattice points.

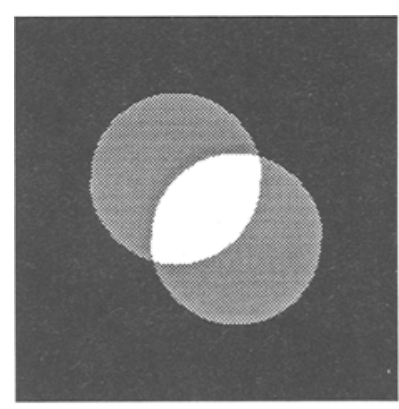

(a) Single.

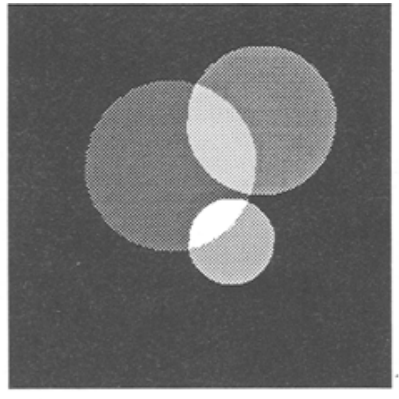

(b) Multiple.

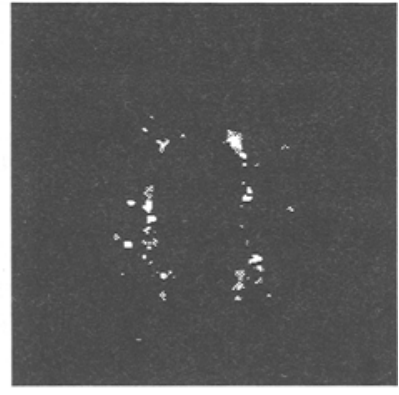

(c) MS lesions (2-D).

Fig. 1. Sample synthetic objects and MS lesions. Brighter areas indicate elements common to both images. 


\subsection{Single Objects}

Synthetic objects as those in Fig. 1.a were generated and individually labelled. The size of the objects $(b, c)$, their overlap $(a)$ and the size of the lattice $(Q)$ were varied to observe the response of $C_{j k}$ and $C_{k j}$. Fig. 2.a shows the behaviour of $C_{j k}$ when both objects have the same size and their degree of overlap is varied from $0.01 \%$ to $100 \%$. The different curves correspond to increases in $Q$. For the bottom curve $Q=b+c$ and for the top curve $Q=b * c$.

\subsection{Multiple Objects}

The experiments to study the behaviour of $C_{j}$ and $C_{k}$ consisted of generating a large single object in the observation image and two small objects in the reference. Their degree of overlap $(a=b \cap c, e=b \cap f)$ was simultaneously varied, keeping their size constant and using a lattice size $Q=b+c+f$. Fig. 2.b presents the response of $C_{j}$, the spatial correspondence between $b$ and the objects $c$ and $f$. When segmenting MS lesions in MR scans this situation occurs if an observer subdivides a large lesion identified as a single lesion by the other observer. Because the total area of both segmentations is the same, $C_{j}$ is an estimate of the boundary placement error.

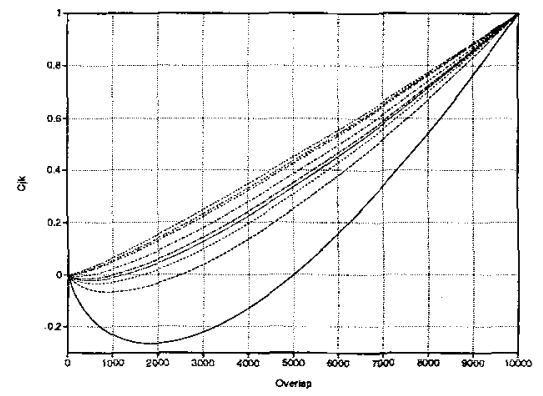

(a) $\mathrm{b}=\mathrm{c}=10000$

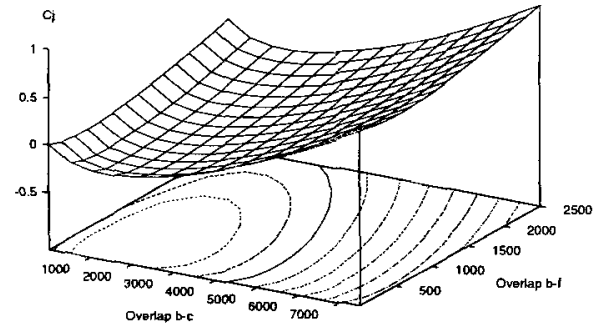

(b) $b=10000, c=7500, f=2500$

Fig. 2. Behaviour of $C_{j k}$ and $C_{j}$.

\subsection{MS Lesions}

To evaluate the performance of the new indices in MS, two 3-D $T_{2}$-weighted MR brain scans from different MS patients were segmented by two observers. In both cases there was significant disagreement in the total number of lesions (Case 1: $K=145, J=581$; Case 2: $K=113, J=278$ ), but the total lesion volume was significantly different in only one of the cases (Case 1: $V o l_{K}=16.49 \mathrm{ml}$, $V o l_{J}=25.69 \mathrm{ml} ;$ Case $\left.2: V l_{K}=13.39 \mathrm{ml}, V_{o l}=14.96 \mathrm{ml}\right)$. Fig. 3 shows surface projections of the segmentations done by Observer-1 $\left(X_{k}\right)$ and Observer-2 $\left(Y_{j}\right)$. After uniquely labelling all the lesions in each segmentation, a Correspondence matrix was formed and percentage measures of individual (one-to-one), local 
(many-to-one) and global (all-to-all) spatial correspondences were computed. For simplicity, the 3-D image grid was used as the lattice $\boldsymbol{G}$.

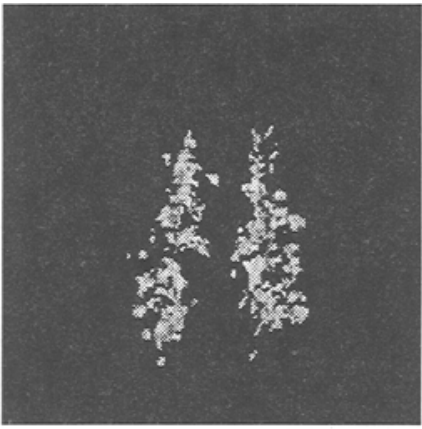

(a) Case 1 - Observer-1

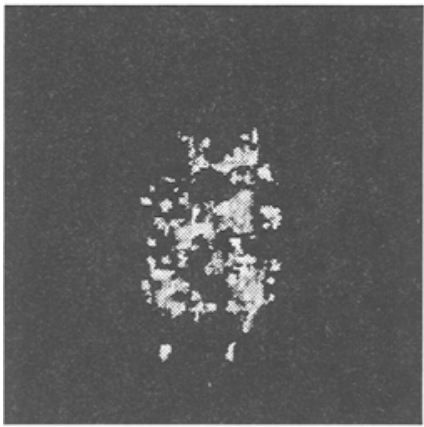

(c) Case 2 - Observer-1.

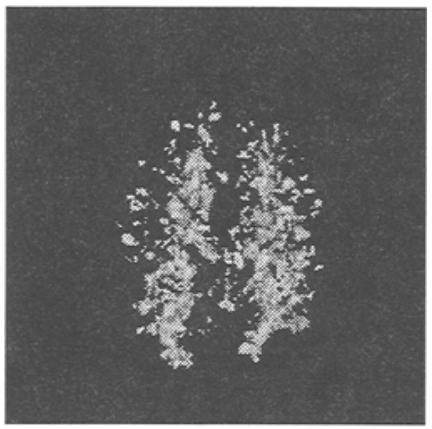

(b) Case 1 - Observer-2

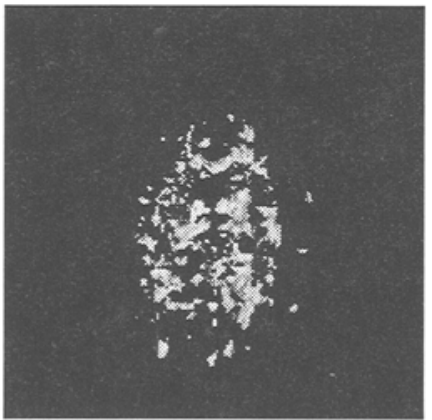

(d) Case 2 - Observer-2.

Fig. 3. Surface rendering of segmented MS lesions.

Table 1 shows the values of the individual spatial correspondence measures between a lesion from Case 1 segmented by Observer-2 $\left(Y_{93}\right)$ and all the lesions segmented by Observer-1 with which it overlaps $\left(X_{19}, X_{34}, X_{45}, X_{62}, X_{86}, X_{92}\right.$, $X_{94}, X_{95}$ and $\left.X_{113}\right)$.

\begin{tabular}{|c|c|c|c|c|c|c|c|c|c|}
\hline \multicolumn{2}{|c|}{ Observer-2 } & \multicolumn{2}{|c|}{ Observer-1 } & \multirow[t]{2}{*}{$X_{k} \cap Y_{j}$} & \multirow[t]{2}{*}{$C_{k j}$} & \multirow[t]{2}{*}{$C_{j k}$} & \multirow[t]{2}{*}{$\operatorname{Err} A$} & \multirow[t]{2}{*}{ Over } & \multirow[t]{2}{*}{ Sim } \\
\hline$j$ & Voxels & $k$ & Voxels & & & & & & \\
\hline \multirow{9}{*}{93} & \multirow{9}{*}{1530} & 19 & $\overline{52}$ & $\overline{42}$ & 2.66 & 54.58 & -86.85 & 2.72 & 5.30 \\
\hline & & 34 & 28 & 28 & 1.83 & 65.83 & -92.81 & 1.83 & 3.59 \\
\hline & & 45 & 499 & 349 & 21.75 & 58.23 & -1.62 & 20.77 & 34.40 \\
\hline & & 62 & 34 & 19 & 1.14 & 34.58 & \begin{tabular}{|l|}
-91.30 \\
\end{tabular} & 1.22 & 2.42 \\
\hline & & 86 & 20 & 14 & 0.87 & 42.72 & \begin{tabular}{|l|}
-94.83 \\
\end{tabular} & 0.91 & 1.80 \\
\hline & & 92 & 422 & 270 & 16.62 & 51.64 & -13.52 & 16.05 & 27.66 \\
\hline & & 94 & 3 & 3 & 0.19 & 55.28 & -99.21 & 0.19 & 0.39 \\
\hline & & 95 & 4 & 4 & 0.26 & 56.45 & -98.95 & 0.26 & 0.52 \\
\hline & & 113 & 36 & 33 & 2.13 & 60.97 & -90.80 & 2.15 & 4.21 \\
\hline
\end{tabular}

Table 1. Measures of spatial correspondence between individual lesions. 
The local spatial correspondence for the above example of lesion subdivision is presented in Table 2, where a local overlap index and a local similarity index have been computed by adding all the individual measures.

\begin{tabular}{|r||r|r|r|}
\hline Lesion & $C_{j}$ & Over & Sim \\
\hline \hline$Y_{93}$ & 47.45 & 46.1 & 80.29 \\
\hline
\end{tabular}

Table 2. Measures of local spatial correspondence for lesion $Y_{93}$.

Lastly, Table 3 shows the values of the global spatial correspondence measures for the two cases under consideration.

\begin{tabular}{|c|c|c|c||c|c|c|c|c|}
\hline Case & Voxels in $X$ & Voxels in $Y$ & Voxels in $X \cap Y$ & $C_{X}$ & $C_{Y}$ & Err A & Over & Sim \\
\hline \hline 1 & 8172 & 12735 & 5761 & 20.28 & 39.83 & 56.34 & 38.03 & 55.11 \\
\hline 2 & 6636 & 7418 & 4927 & 57.07 & 44.37 & 88.87 & 53.98 & 70.11 \\
\hline
\end{tabular}

Table 3. Measures of global spatial correspondence.

\section{Results and Discussion}

To better understand the characteristics of the new indices and the results of the previous experiments we can express Eq. 1,3 and 5 in terms of the number of elements in $X_{k}, Y_{j}$ and $X_{k} \cap Y_{j}$ :

$$
\begin{gathered}
C_{j k}=\frac{X_{k} \bigcap Y_{j}}{X_{k}} \cdot \frac{I_{X ; Y}(k, j)}{I_{X}(k)} \\
C_{j}=\sum_{k=1}^{K} \frac{X_{k} \bigcap Y_{j}}{Y_{j}} \cdot \frac{I_{X ; Y}(k, j)}{I_{Y}(j)} \\
C_{\boldsymbol{Y}}=\frac{\sum_{k=1}^{K} \sum_{j=1}^{J} X_{k} \bigcap Y_{j} \cdot I_{X ; Y}(k, j)}{\sum_{k=1}^{K} X_{k} \cdot I_{X}(k)}
\end{gathered}
$$

It is then possible to interpret the new indices as a weighted ratio of the number of overlapping elements to the total number of elements in the reference object. The weighting factor is an uncertainty coefficient that indicates the fraction of the information in the observation that is redundant in the reference. The value of this factor depends not only on the degree of overlap between the objects, but also on the size of the sampling lattice $\boldsymbol{G}$. This dependence of the new indices on the size of the sampling lattice is clearly shown in Fig. 2.a.

Considering $\boldsymbol{G}$ as the space of possible disagreement between the observers, if its size is small relative to the size of the objects, the proposed measures will only be greater than 0 if the overlap between the objects is larger than that expected by mere chance $\left(P_{X Y}(k, j)>P_{X}(k) P_{Y}(j)\right)$. If we let $Q$, the size of the lattice, increase arbitrarily, Equations 7, 8 and 9 are reduced to the following: 


$$
\begin{gathered}
\lim _{Q \rightarrow \infty} C_{j k}=\frac{X_{k} \cap Y_{j}}{X_{k}} \\
\lim _{Q \rightarrow \infty} C_{j}=\sum_{k=1}^{K} \frac{X_{k} \cap Y_{j}}{Y_{j}} \\
\lim _{Q \rightarrow \infty} C_{\boldsymbol{Y}}=\frac{\sum_{k=1}^{K} \sum_{j=1}^{J} X_{k} \cap Y_{j}}{\sum_{k=1}^{K} X_{k}}
\end{gathered}
$$

In this situation of maximum uncertainty where $\lim _{Q \rightarrow \infty} I_{X ; Y}(k, j)=\infty$ and $\lim _{Q \rightarrow \infty} I_{X}(k)=\infty$, the new indices will always be positive as any degree of overlap, however small, will be larger than that expected by mere chance.

Regarding the size of the objects, $C_{j k}$ will be equal to $C_{k j}$ only when both objects are of the same size. This non-symmetric nature manifests the intuitive non-reflexive relationship between a large object and a small object.

The effect of considering the space of possible disagreement and the nonsymmetric nature of the new indices becomes evident when comparing them with the more established measures. If the lattice size is small relative to the size of the object, the values of $C_{j k}$ and $C_{k j}$ are well below those of the other measures. This is to be expected as the new indices will penalise large errors when the space of possible disagreement is small. As the lattice size is increased, $C_{j k}$ and $C_{k j}$ tend to bound the values of the overlap index and the similarity index. If the objects are of the same size $\left(C_{j k}=C_{k j}\right)$, then $\lim _{Q \rightarrow \infty} C_{j k}=\operatorname{Sim}$. If the objects have different size and the smaller object (e.g. $Y_{j}$ ) is fully enclosed by the large object (e.g. $X_{k}$ ), then $C_{j k}=$ Over.

Table 1 demonstrates the behaviour of the individual correspondence indices for various combinations of lesion size and overlap. The non-reflexive relationship between the larger 'inclusive' object and the smaller 'split' objects is clearly reflected in the different values obtained for $C_{j k}$ and $C_{k j}$. The relative values of $C_{j k}$ and $C_{k j}$ are a measure of the boundary placement error. The larger the difference in their values, the larger the error. The absolute values of $C_{j k}$ and $C_{k j}$, on the other hand, quantify the agreement in lesion identification. The larger the values, the more likely it is that the lesions identified by the observers are the same. With this in mind, for the sample lesions in Table 1 it can be said that there is a very large boundary placement error and that, apart from lesions $X_{45}$ and $X_{92}$, the lesions segmented by Observer-1 should probably be interpreted as completely different lesions with respect to $Y_{93}$. Comparing the new indices with the other measures in this table, the values of $C_{k j}$ are close to those of the overlap index because all of the objects $X_{k}$ are smaller than the object $Y_{j}$ and are partly or fully enclosed by it. $C_{j k}$, on the other hand, shows significantly larger values reflecting the fact that a larger proportion of the elements in each of the small objects $X_{k}$ is included in the large object $Y_{j}$. The values of the similarity index are larger than those of $C_{k j}$, but are still bounded by $C_{j k}$. Regarding the complement area error, its values suggest that it is not well suited to estimate the correspondence when there are large 
differences in size between the objects. None of these three measures can be used to further study the similarities and differences between the observers as is the case with $C_{j k}$ and $C_{k j}$.

An example of applying the local spatial correspondence measure $C_{j}$ to MS is shown in Table 2, where part of the volume segmented by Observer-2 as lesion $Y_{93}$ has been identified as nine separate lesions by Observer-1. From the analysis above, this can be considered as an extreme case of boundary placement disagreement between the observers. $C_{j}$ is a measure of the local agreement between the observers that is influenced by both the boundary placement error and the lesion identification error. Large values of $C_{j}$ indicate that there is agreement between the observers as to the location and possible extent of a certain lesion area, even if one of the observers has subdivided the lesion area in various regions. The value of $C_{j}$ is slightly larger than the local overlap index and much smaller than the local similarity index, which seems to significantly overestimate the local spatial correspondence.

Lastly, Table 3 presents the global spatial correspondence indices for both examples under consideration. In this case, the relative values of $C_{\boldsymbol{X}}$ and $C_{\boldsymbol{Y}}$ are related to the boundary placement and the lesion identification errors. The larger the difference in their values, the larger the difference in the total number of lesions and the total lesion volume segmented by the observers. The absolute values of $C_{\boldsymbol{X}}$ and $C_{\boldsymbol{Y}}$, on the other hand, are a measure of the overall spatial correspondence between the observers. As expected, $C_{\boldsymbol{X}}$ and $C_{\boldsymbol{Y}}$ are larger for Case 2 (large difference in total number of lesions, but small difference in total volume) than for Case 1 (large difference in total number of lesions and in total volume). This is also true for the other three measures, but closer examination of the new indices also reveals that in Case 1 the area common to both observers has a greater correspondence with the segmentation done by Observer-1 $\left(C_{\boldsymbol{Y}}>C_{\boldsymbol{X}}\right)$, whereas in Case 2 the area common to both observers has a greater correspondence with the segmentation done by Observer-2 $\left(C_{\boldsymbol{X}}>C_{\boldsymbol{Y}}\right)$. This reflects an inconsistency between the observers which is not at all apparent from any of the other measures. Moreover, the complement area error and the similarity index tend to overestimate the spatial correspondence, whereas the values of the overlap index are once again bounded by the new indices.

\section{Conclusions and Future Work}

In this paper we introduced the concept of a Correspondence Matrix that can be used to compute measures of spatial correspondence between binary objects segmented by two different observers and/or segmentation algorithms. Using Information Theory we then derived a set of indices to estimate individual (oneto-one), local (many-to-one) and global (many-to-many) spatial correspondence. The main characteristics of the new indices are the implicit consideration of the space of possible disagreement and their non-symmetric nature.

The behaviour of the new indices was shown to be consistent for both synthetic and real objects regardless of their number and size. Comparing the new 
individual spatial correspondence indices with the more established measures of similarity illustrated the effect of considering the space of possible disagreement, and their ability to distinguish and quantify the boundary placement and lesion identification errors as a result of their non-symmetric nature. At a local level, the new indices showed the importance of quantifying the correspondence between subdivided objects and the risk of overestimating such correspondence. Globally, the proposed indices proved to be sensitive to the overall spatial correspondence as well as to more subtle inconsistencies between the observers.

In general, the values of the new indices act as bounds to those obtained for the overlap index, while the similarity index seems to consistently overestimate spatial correspondence and the complement area error is either not well suited or overestimates spatial correspondence. This possible overestimation and the proved lack of sensitivity of these three measures to quantify errors and inconsistencies make the proposed new indices highly attractive for medical imaging applications, such as MS lesion segmentation, where validation and comparison of segmentation results obtained by different observers and/or automated algorithms is vital.

Amongst the further work under consideration is the determination of the optimal size of the sampling lattice $\boldsymbol{G}$, the use of thresholds to establish the number of corresponding and non-corresponding lesions, and the development of a methodology based on the new indices to automatically quantify spatial correspondence and spatial dissimilarity through time or across modalities.

\section{Acknowledgements}

This work is part of BIOMORPH (Development and Validation of Techniques for Brain Morphometry), project PL95 0845 of the BIOMED programme of the European Commission. Special thanks go to Prof. R. Hughes and Dr. B. Sharrack from the Department of Neurology at UMDS-Guy's Hospital for providing the MR data and the MS lesion segmentations.

\section{References}

1. Schalkoff, R. Pattern Recognition. J. Wiley \& Sons, 1992.

2. Zidjenbos, A P; Dawant, B M; Margolin, R A and Palmer A C. Morphometric Analysis of White Matter Lesions in MR Images: Method and Validation. IEEE T.M.I. 13:716-724, 1994.

3. Brummer, M E; Mersereau, E M; Eisner, R L and Lewine, R R J. Automatic Detection of Brain Contours in MRI Data Sets. IEEE T.M.I. 12:153-166, 1993.

4. Grimaud J; Lai M; Thorpe J; Adeleine P; Wang G and Barker G J. Quantification of MRI Lesion Load in Multiple Sclerosis: a comparison of Three Computer-assisted Techniques. Magnetic Resonance Imaging 14:495-505, 1996.

5. Jackson E F; Narayana P A; Wolinsky J S and Doyle T J. Accuracy and Reproducibility in Volumetric Analysis of Multiple Sclerosis Lesions. Journal of Computer Assisted Tomography 17:200-205, 1993.

6. Wells, W M; Viola, P; Atsumi, H; Nakajima, S and Kikinis, R. Multi-modal Volume Registration by Maximization of Mutual Information. Medical Image Analysis 1:35-51, 1996.

7. Maes, F; Collignon, A; Vandermeulen, D; Marchal, G and Suetens, P. Multimodality Image Registration by Maximization of Mutual Information. IEEE T.M.I. 16:187-198, 1997.

8. Weaver, W and Shannon, C E. The Mathematical Theory of Communication. Univ. of Illinois Press, 1949. 